\title{
Ultra-short-pulse high-average-power megahertz-repetition-rate coherent extreme-ultraviolet light source
}

\author{
Robert Klas ${ }^{1,2^{*}} \mathbb{D}$, Alexander Kirsche ${ }^{1,2}$, Martin Gebhardt ${ }^{1,2}$, Joachim Buldt ${ }^{1}$, Henning Stark ${ }^{1}$, Steffen Hädrich ${ }^{3}$,
} Jan Rothhardt ${ }^{1,2,4}$ and Jens Limpert ${ }^{1,2,4}$

\author{
*Correspondence: robert.klas@uni- \\ jena.de \\ ${ }^{1}$ Institute of Applied Physics, Abbe \\ Center of Photonics, \\ Friedrich-Schiller-University Jena, \\ Albert-Einstein-Str. 15, 07745 Jena, \\ Germany \\ ${ }^{2}$ Helmholtz-Institute Jena, \\ Fröbelstieg 3, 07743 Jena, Germany \\ Full list of author information is \\ available at the end of the article
}

\begin{abstract}
High harmonic generation ( $\mathrm{HHG}$ ) enables coherent extreme-ultraviolet (XUV) radiation with ultra-short pulse duration in a table-top setup. This has already enabled a plethora of applications. Nearly all of these applications would benefit from a high photon flux to increase the signal-to-noise ratio and decrease measurement times. In addition, shortest pulses are desired to investigate fastest dynamics in fields as diverse as physics, biology, chemistry and material sciences. In this work, the up-to-date most powerful table-top XUV source with $12.9 \pm 3.9 \mathrm{~mW}$ in a single harmonic line at $26.5 \mathrm{eV}$ is demonstrated via HHG of a frequency-doubled and post-compressed fibre laser. At the same time the spectrum supports a Fourier-limited pulse duration of sub- 6 fs in the XUV, which allows accessing ultrafast dynamics with an order of magnitude higher photon flux than previously demonstrated. This concept will greatly advance and facilitate applications of XUV radiation in science and technology and enable photonhungry ultrafast studies.
\end{abstract}

\section{Introduction}

Since the first experimental demonstration of high harmonic generation in the late 1980s [1, 2], strong efforts have been made to enhance the average power of laser-like sources in the XUV [3], enabling applications on the atomic length-(nanometer) [4] and time-scale (femtosecond to attosecond) [5, 6]. In the early stages up to the year 2010 (Fig. 1), Ti:Sa based amplifiers have been proven as an effective driver for HHG $[3,7,8]$, since they provide ultra-short pulse durations $(\sim 25 \mathrm{fs})$ at $800 \mathrm{~nm}$ wavelength and high pulse energies (several millijoule). However, their limited average power of a few tens of watts in best case [16], limited the XUV flux of such systems to sub$100 \mu \mathrm{W}$ per harmonic line (Fig. 1). An increase in XUV average power would help for example to mitigate space charge effects in photoelectron emission spectroscopy [17] (at high repetition rates), as well as to shorten acquisition times and, hence, enhance the signal-to-noise ratio in (time-resolved) coincidence measurements [18], XUVabsorption spectroscopy [19], XUV-ionization spectroscopy [20], coherent diffractive imaging of ultrafast magnetization dynamics [21], fluorescence spectroscopy [22] and

(c) The Author(s). 2021 Open Access This article is licensed under a Creative Commons Attribution 4.0 International License, which permits use, sharing, adaptation, distribution and reproduction in any medium or format, as long as you give appropriate credit to the original author(s) and the source, provide a link to the Creative Commons licence, and indicate if changes were made. The images or other third party material in this article are included in the article's Creative Commons licence, unless indicated otherwise in a credit line to the material. If material is not included in the article's Creative Commons licence and your intended use is not permitted by statutory regulation or exceeds the permitted use, you will need to obtain permission directly from the copyright holder. To view a copy of this licence, visit http://creativecommons.org/licenses/by/4.0/. 


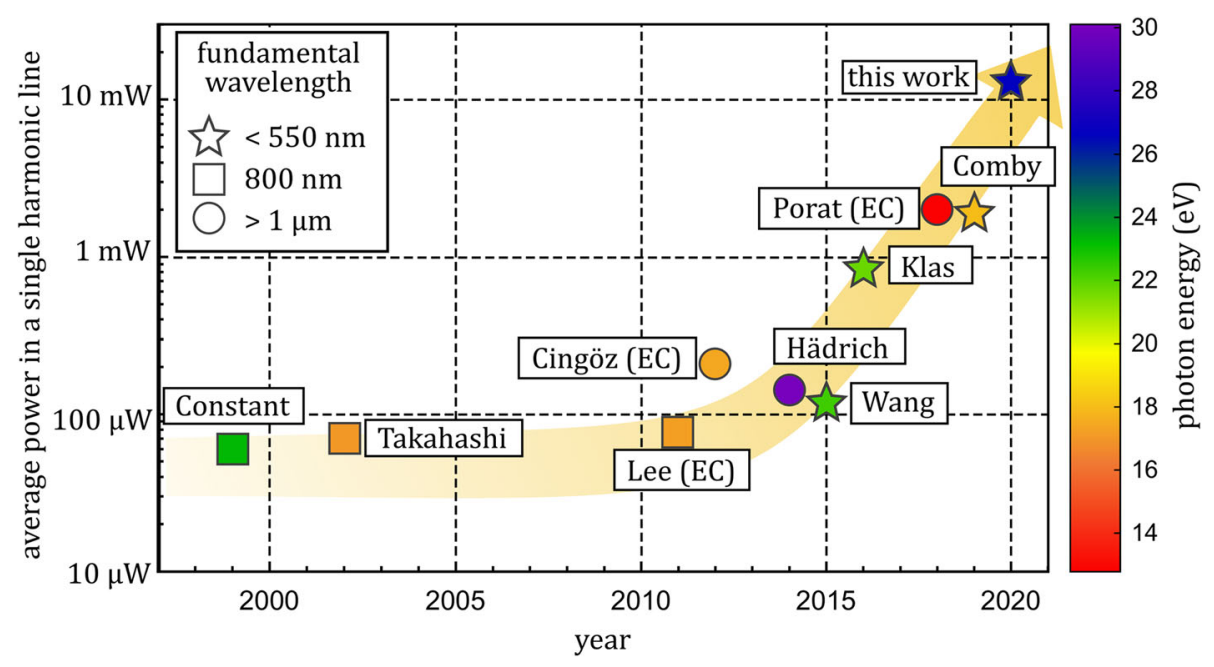

Fig. 1 State of the art high harmonic sources. Assorted sources with, at the time, record high average powers above $10 \mu \mathrm{W}$ in a single harmonic line for photon energies ranging from $12 \mathrm{eV}$ to $30 \mathrm{eV}$ over a time span from 1999 until to date [7-15]. The photon energy is indicated by the marker colour, while the driving wavelength is indicated by the marker shape. Squares are for Ti:Sa based systems with a wavelength of 800 $\mathrm{nm}$, circles are for Yb-based systems at $>1 \mu \mathrm{m}$ wavelength and stars are for cascaded schemes for high harmonic generation with the second or third harmonic of the prior mentioned architectures. (EC) marks sources based on an enhancement cavity, while all other sources are in a single pass geometry

XUV-pump XUV-probe experiments [23, 24], among others. Furthermore, shortest pulses are desired to investigate fastest dynamics in atoms [6,25], molecules [26], ions [23], solids [19] and compound materials [27].

Inherently, an increase in XUV average power can be achieved with high average power driving lasers. In recent years, enhancement cavities $[9,10]$ as well as Yb-based fibre lasers [11], which are capable of much higher average powers in the kilowatt regime (enabling megahertz repetition rates [28]), have shown a first increase in XUV average power demonstrating $>100 \mu \mathrm{W}$ in a single harmonic line [10, 11], with typical conversion efficiencies $<10^{-5}$. Further increase of the HHG efficiency and, hence, XUV average powers in the milliwatt-regime can be achieved by using a combination of an optimized enhancement cavity together with a high average power driving laser [12], or by using short wavelength drivers $[13,14]$. The latter ones made use of the efficiency scaling of the single atom response with $\lambda^{6}$, where $\lambda$ represents the driving wavelength [29], increasing the HHG efficiency by more than one order of magnitude.

Shorter driving pulses can enhance the efficiency and the cutoff energy of the XUV comb even more and naturally generate shorter pulses in the XUV. This can be understood since higher intensities $I$ can be applied for phase-matched HHG [3]. Furthermore, the macroscopic yield scales with the square of the single atom dipole moment $A_{q}$ [7], which increases with increasing intensity [7, 30-32]. In this manuscript the model by Lewenstein et al. is used [32], resulting in higher efficiencies for shorter pulses and a $~ 3$ times shorter XUV pulse duration (Supplement).

However, two-photon absorption in high-reflective and anti-reflective coatings as well as in transmission optics, and the consequential heating of the materials, makes it very challenging to generate high average power as well as ultra-short pulse duration driving lasers with $<550 \mathrm{~nm}$ wavelength. Therefore, until now, short wavelength driven HHG 
with milliwatt XUV average power was done using $>85 \mathrm{fs}$ pulses and average powers of a few Watt $[12,13]$.

In this letter, HHG driven by the unique combination of high average power (51 W), short driving wavelength $(515 \mathrm{~nm})$ and ultra-short pulse duration $(18.6 \mathrm{fs})$ is demonstrated. These ultrashort visible pulses enable the generation of XUV pulses with a conversion efficiency of $2.5 \cdot 10^{-4}$, and, consequently the high average power of the driving laser boosts the photon flux to a record of $12.9 \pm 3.9 \mathrm{~mW}$ in a single harmonic line at $26.5 \mathrm{eV}$ - surpassing previously demonstrated sources by an order of magnitude (Fig. 1).

\section{Results and discussion}

\section{Pulse compression at high average power and $515 \mathrm{~nm}$ wavelength}

For the experiment performed herein, a $515 \mathrm{~nm}$ laser at a repetition rate of $1 \mathrm{MHz}, 89$ $\mathrm{W}$ of average power and $200 \mathrm{fs}$ is compressed, using a nonlinear hollow core fibre compressor. Careful selection of UV-grade fused silica glasses and coating materials for transmission and reflection optics, that have a low two-photon absorption and a large $\mathrm{OH}$-content of up to $1000 \mathrm{ppm}$, make it possible to handle such high average powers at $515 \mathrm{~nm}$. Simulations to optimize the output peak power while maintaining a low ionization level, revealed an optimal fibre diameter of $150 \mu \mathrm{m}$ at a fixed length of $1 \mathrm{~m}$ (Methods). Spectral broadening, shown in Fig. 2a), is achieved by filling such a capillary with 0.8 bar krypton. The transmission of $59 \%$ is the same for an evacuated as well as a gas-filled capillary, showing that no significant ionization is present. Temporal compression of the spectrally broadened pulses is done using a custom designed chirped mirror compressor, which shows virtually no heating of the chirped mirrors due to

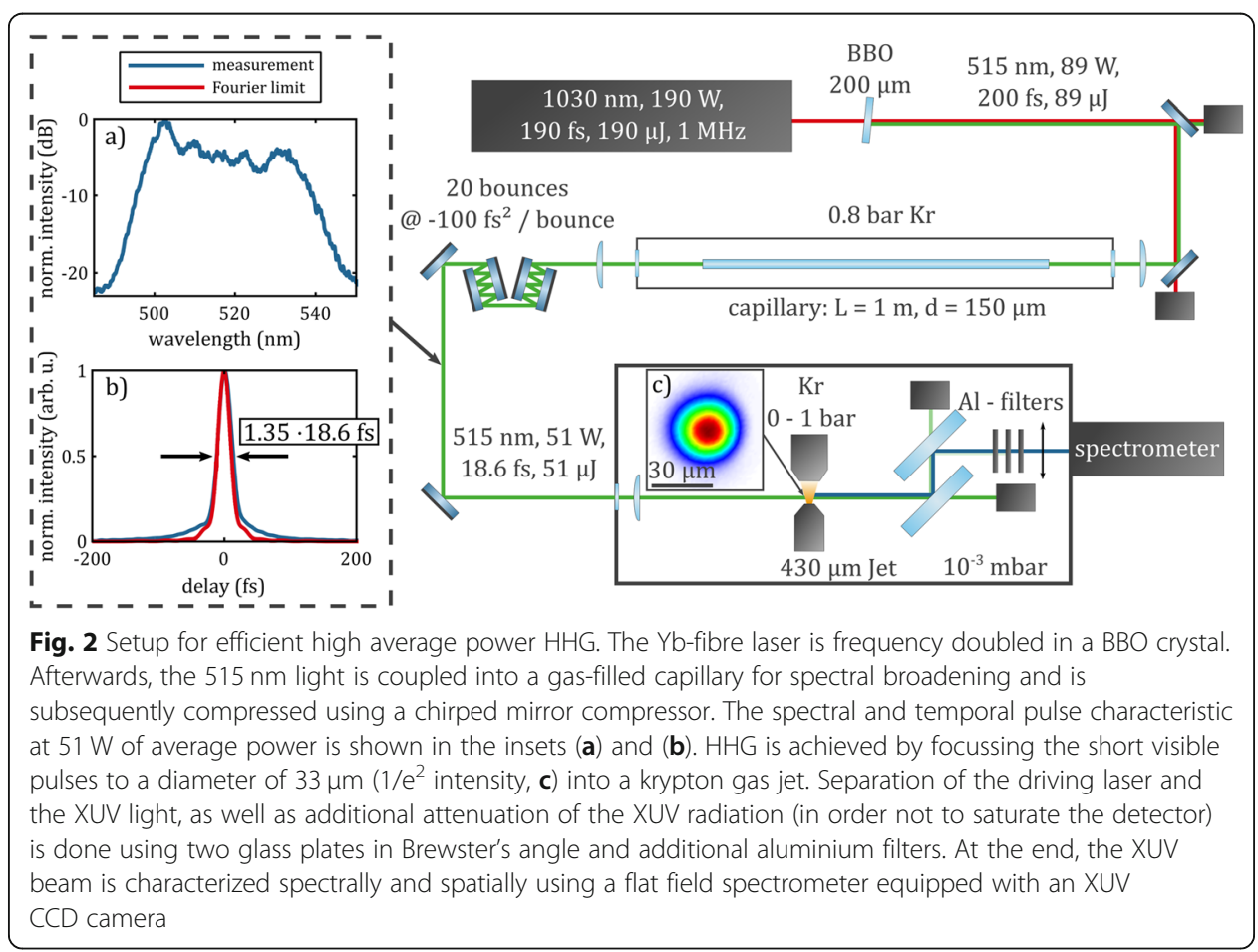


their high reflectivity, resulting in a transmission of $96 \%$. The overall dispersion is $2000 \mathrm{fs}^{2}$ ( 20 bounces on $-100 \mathrm{fs}^{2}$ /bounce mirrors), which is also compensating the glass dispersion of the used lenses and windows of $1200 \mathrm{fs}^{2}$. Fourier transforming the broadened spectrum (Fig. 2a)) reveals a deconvolution factor for the autocorrelation of 1.35, uncovering a compressed pulse duration of $18.6 \mathrm{fs}$ (Fourier limit $16.5 \mathrm{fs}$ ) at a record average power of $51 \mathrm{~W}$ and a pulse energy of $51 \mu \mathrm{J}$, resulting in a peak power of $>2.5$ GW. Furthermore, a spatial characterization of this compressed beam shows a nearly diffraction limited beam quality with an $\mathrm{M}^{2}$ value of $1.25 \times 1.26$. This unique combination of short wavelength, ultrashort pulse duration, high average power and very good beam quality represents a novel class of driving laser for high harmonic generation at a five-fold higher average power compared to previous results [33, 34].

\section{High harmonic generation results}

HHG is achieved by focussing these pulses to a diameter of $33 \mu \mathrm{m}\left(1 / \mathrm{e}^{2}\right.$ intensity, Fig. 2c)) into a krypton gas jet. The generated harmonics and the remaining visible light pass through two glass plates in Brewster's angle and various $1 \mu \mathrm{m}$ thick aluminium filters, not only to separate the higher average power driving laser from the generated extreme ultraviolet light, but also to attenuate the XUV light for further analysis. Subsequently, a flat field spectrometer equipped with a CCD camera is used to analyse the XUV beam spectrally and spatially. Optimization of the XUV flux is done by iteratively optimizing the nozzle position on a 3D-translation stage and an iris in front of the vacuum chamber. The optimal phase-matching pressure is found via variation of the applied pressure, revealing an optimum at $0.4 \mathrm{bar}$. The result of a corresponding simulation with a one dimensional model support these findings, assuming a distance between the laser beam and the nozzle of $144 \mu \mathrm{m}$ (supplement). This results in a particle density in the interaction region of $3.8 \cdot 10^{18} \mathrm{~cm}^{-3}$ and an absorption length of $130 \mu \mathrm{m}$ at $26.5 \mathrm{eV}$. Consequently, the medium length defined by the nozzle diameter $(430 \mu \mathrm{m})$, which is much shorter than the Rayleigh length $(1.3 \mathrm{~mm})$, allows for absorption-limited HHG [7].

Due to the ultra-short pulse duration at $515 \mathrm{~nm}$, the HHG efficiency into a single harmonic line at $26.5 \mathrm{eV}$ is $2.5 \cdot 10^{-4}$, which is among the highest reported so far [8, 14]. The short pulse duration is important for this high efficiency since higher intensities can be applied for phase-matched HHG, compared to previous experiments using 85 fs driving laser pulses [13], the shorter driving laser pulses show a threefold increase in efficiency as well as a $>5 \mathrm{eV}$ higher cut-off energy. Furthermore, the high average power $(51 \mathrm{~W})$ of the driving laser allows $23.1 \mathrm{~mW}$ of average power in the range from $20 \mathrm{eV}$ to $35 \mathrm{eV}$ (Fig. 3a)), with a record high average power of $12.9 \pm 3.9 \mathrm{~mW}$ in the strongest harmonic line at $26.5 \mathrm{eV}$. The relative uncertainty is estimated conservatively to be $<30 \%$, with the main contributions originating from the measured filter transmission (7\%) and the grating efficiency (20\%). In addition, the increased cutoff allows for $>$ $1 \mathrm{~mW}$ of average power above $30 \mathrm{eV}$. Note, that for a high flux delivery, a gracing incidence plate [35] or annular beam driven HHG [36] in combination with a $100 \mathrm{~nm}$ aluminium filter could be used as a separator, resulting in an attenuation of the driving laser to $<1 \mu \mathrm{W}$ with $>5 \mathrm{~mW}$ usable XUV average power on target. The Fourier-limited pulse duration of a single harmonic line corresponds to $3.4 \mathrm{fs}$ pulse duration in the 
a)

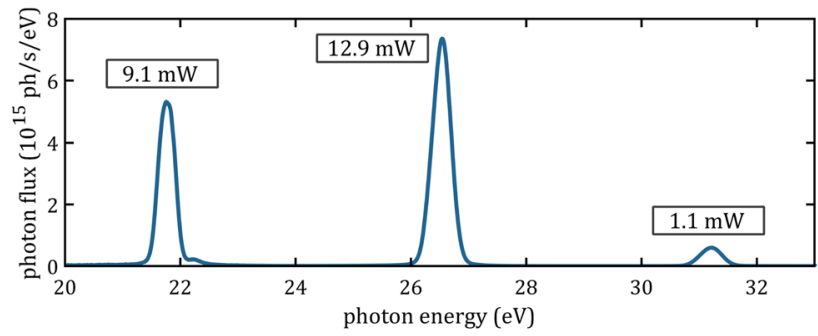

c)

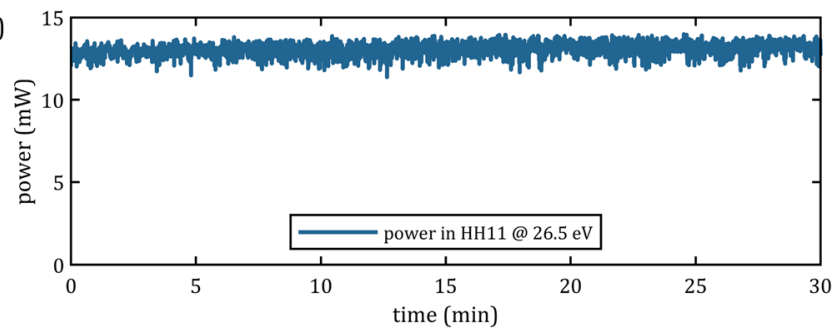

b)
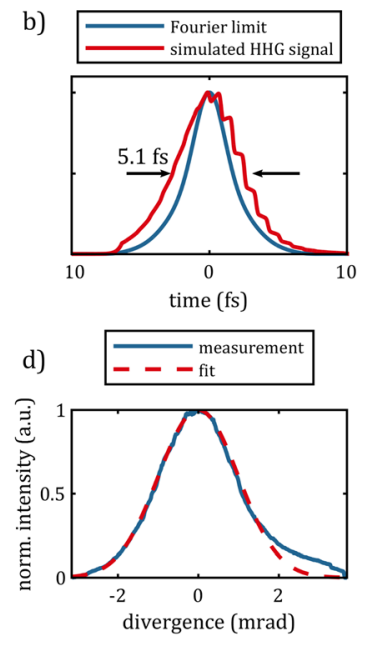

Fig. 3 High harmonic generation at $1 \mathrm{MHz}$. a Generated high harmonic spectrum using krypton with a backing pressure of 0.4 bar and a $430 \mu \mathrm{m}$ gas nozzle, together with the corresponding average power in each harmonic line. The highest average power in the 11th harmonic of $12.9 \pm 3.5 \mathrm{~mW}$ corresponds to a photon flux of $3 \cdot 10^{15} \mathrm{ph} / \mathrm{s}$. b Fourier limited pulse duration of the 11 th harmonic at $26.5 \mathrm{eV}$ of $3.4 \mathrm{fs}$ (FWHM) as well as the simulated high harmonic signal (5.1 fs, supplement). $\mathbf{c}$ Long term measurement of the average power in the 11th harmonic at $26.5 \mathrm{eV}$ over a time period of 30 min every $0.2 \mathrm{~s}$, showing an rms deviation of $2.6 \%$. d Spatial lineout of the whole XUV beam with a corresponding Gaussian fit

XUV (Fig. 3b)), which is in good agreement with the duration of the simulated phasematching window (5.1 fs, supplement). Focussing these pulses to a spot size of $1 \mu \mathrm{m}$, would result in an intensity $>10^{14} \mathrm{~W} / \mathrm{cm}^{2}$. Thus, even nonlinear XUV-techniques are in reach at a $\mathrm{MHz}$ repetition rate. A long-term stability measurement of the photon flux of the strongest harmonic line at $26.5 \mathrm{eV}$ shows an rms-deviation of $2.6 \%$ over a time period of $30 \mathrm{~min}$ (Fig. 3c)). Furthermore, a spatial lineout integrated over the whole spectrum together with a Gaussian fit shows a Gaussian like beam profile of the XUV beam with a divergence of $2 \mathrm{mrad}$ (Fig. 3d)), showing the great potential of this source for further experiments.

\section{Conclusion}

In conclusion, a high power XUV source via HHG, delivering an average power of $12.9 \pm 3.9 \mathrm{~mW}$ in single harmonic line at $26.5 \mathrm{eV}$ and $>1 \mathrm{~mW}$ at photon energies above $30 \mathrm{eV}$ is demonstrated. Furthermore, the spectrum supports a Fourier limited pulse duration of $3.4 \mathrm{fs}$ and simulation results suggest that the time span of the efficient high harmonic generation is shorter than 6 fs. This record XUV power together with the cutoff enhancement is enabled by a new-class of driving laser providing a unique combination of a short wavelength $(515 \mathrm{~nm})$ and a short pulse duration $(18.6 \mathrm{fs})$ at a record high average power of $51 \mathrm{~W}$. Compared to state-of-the art XUV sources, the provided average power is one order of magnitude higher and the pulse duration is significantly shorter, which represents a major milestone for upcoming applications of coherent $\mathrm{XUV}$ radiation in science and technology. Especially compared to $\mathrm{Yb}$-fiber laser driven HHG sources the overall system efficiency from the Yb-fiber CPA into the XUV is 7 . $10^{-5}$, which is the highest efficiency ever shown for fiber laser driven HHG sources [10-14]. This will greatly advance and facilitate XUV application in various fields e.g. 
investigating fastest dynamics using photoelectron emission spectroscopy, coincidence measurements, XUV- ionization and absorption spectroscopy, fluorescence spectroscopy, ultrafast XUV imaging and XUV-pump XUV-probe experiments, among others [17-24, 26, 27]. Due to the excellent scaling possibilities of energetic ultrafast Yb-based fibre lasers and the components for pulse compression to the $\mathrm{kW}$-level and beyond [28, $37,38]$, even upscaling of the presented approach to the $100 \mathrm{~mW}$ level seems possible.

\section{Methods}

Fibre laser setup and second harmonic generation

The high average power $\mathrm{Yb}$-fibre laser system used for the experiments is similar to the one shown by Müller et al. [28]. For the experiments performed herein, four coherently combined amplifier channels are used. At a central wavelength of $1030 \mathrm{~nm}$ and a repetition rate of $1 \mathrm{MHz}$ it delivers a pulse energy of $190 \mu \mathrm{J}$ at a pulse length of $190 \mathrm{fs}$, resulting in $190 \mathrm{~W}$ of average power. This laser is focused into a $200 \mu \mathrm{m}$ thick Type-I beta-barium-borate (BBO) crystal for second harmonic generation (SHG). Using an IR focal size of $590 \mu \mathrm{m}\left(1 / \mathrm{e}^{2}\right.$ intensity) and a resulting intensity of $700 \mathrm{GW} / \mathrm{cm}^{2}$, an SHG efficiency of $47 \%$ is achieved. The resulting $89 \mu \mathrm{J}, 89 \mathrm{~W}$ and $200 \mathrm{fs}$ pulses at $515 \mathrm{~nm}$ have an excellent spatial beam quality with an $\mathrm{M}^{2}$ value of smaller than 1.1 in both axes, as well as a negligible astigmatism.

\section{Hollow core fibre compression simulations}

The numerical simulations of the pulse propagation in the hollow core fibre are performed based on the unidirectional field propagation equation for the fundamental mode [39]:

$$
\partial_{z} E(z, \omega)=i\left(\beta(\omega)-\frac{\omega}{v}\right) E(z, \omega)+i \frac{\omega^{2}}{2 c^{2} \varepsilon_{0} \beta(\omega)} P^{N L}(z, \omega)
$$

with the electric field amplitude in the spectral domain E, the angular frequency $\omega$, the fundamental mode propagation constant $\beta$, the frame reference velocity $v$, the vacuum speed of light $c_{0}$, and the vacuum permittivity $\varepsilon_{0}$. The nonlinear polarization $P^{N L}$ includes the Kerr-nonlinearity and plasma formation (based on Amossov-Delone-Krainov ionization rates [40]).

\section{Characterization of extreme ultraviolet radiation}

The generated photon flux is estimated by accounting for the known efficiencies in the detection apparatus, which are the CCD sensitivity and quantum efficiency ( $1 \mathrm{ph} /$ count), the grating efficiency $(0.11)$, the measured filter transmission $\left(4 \cdot 10^{-4}\right)$, the absorption due to the residual gas (0.7) and theoretical reflectivity of the Brewster plates [41] $\left(6 \cdot 10^{-3}\right.$, which show a good agreement with calibration measurements [13, 35]). The values in the brackets are the correction factors used for the 13th harmonic at $26.5 \mathrm{eV}$. This technique was cross-calibrated using a photodiode (AXUV100G) in various previous experiments and always has shown a good agreement $[11,13]$. 


\section{Supplementary Information}

The online version contains supplementary material available at https://doi.org/10.1186/s43074-021-00028-y.

Additional file 1: This supplementary information provides details on the one-dimensional model used for simulating the high-harmonic build-up. Furthermore, details for the temporal phase-matching as well as for the XUV pulse duration are presented.

\section{Acknowledgements}

Not applicable.

\section{Authors' contributions}

J.L., J.R., S.H. and R. K conceived and planned the experiment. R.K., J.B., H. S, A.K., and M.G. built and optimized the ultrafast fibre laser and the nonlinear compression stage. R.K., A.K., J.B. and H.S. performed the HHG experiments. R.K., S.H. and M. G performed simulations and data analysis. All authors discussed and contributed to the interpretation of the results and to the writing of the manuscript. J.L. and J.R. supervised the project and acquired funding. The author(s) read and approved the final manuscript.

\section{Funding}

This work was supported by the Fraunhofer Cluster of Excellence Advanced Photon Sources (CAPS), by the Innovation Pool of the Research Field Matter of the Helmholtz Association of German Research Centers in project (ECRAPS), by APPA R\&D: Licht- Materie Wechselwirkung mit hochgeladenen lonen (13 N12082), by the Thüringer Ministerium für Bildung, Wissenschaft und Kultur (501100004404, 2017 FGR 0076), by the Thüringer Aufbaubank (TAB Forschergruppe 2015FGR0094), and by the Helmholtz association under grant agreement HGF ExNet-0019-Phase 2-3.

\section{Availability of data and materials}

The datasets used and/or analysed during the current study are available from the corresponding author on reasonable request.

\section{Declarations}

Ethics approval and consent to participate

Not applicable.

\section{Consent for publication}

Not applicable.

\section{Competing interests}

S.H. is employed by Active Fiber Systems who distributes commercial XUV sources.

\section{Author details}

${ }^{1}$ Institute of Applied Physics, Abbe Center of Photonics, Friedrich-Schiller-University Jena, Albert-Einstein-Str. 15, 07745 Jena, Germany. ${ }^{2}$ Helmholtz-Institute Jena, Fröbelstieg 3, 07743 Jena, Germany. ${ }^{3}$ Active Fiber Systems GmbH, Ernst-Ruska-Ring 17, 07745 Jena, Germany. ${ }^{4}$ Fraunhofer Institute for Applied Optics and Precision Engineering, Albert-Einstein-Str. 7, 07745 Jena, Germany.

Received: 19 January 2021 Accepted: 11 April 2021

Published online: 19 April 2021

\section{References}

1. McPherson A, Gibson G, Jara H, Johann U, Luk TS, McIntyre IA, et al. Studies of multiphoton production of vacuumultraviolet radiation in the rare gases. J Opt Soc Am B. 1987;4(4):595. https://doi.org/10.1364/JOSAB.4.000595.

2. Ferray M, L'Huillier A, Li XF, Lompre LA, Mainfray G, Manus C. Multiple-harmonic conversion of $1064 \mathrm{~nm}$ radiation in rare gases. J Phys B Atomic Mol Phys. 1988;21(3):L31-5. https://doi.org/10.1088/0953-4075/21/3/001.

3. Popmintchev T, Chen M-C, Arpin P, Murnane MM, Kapteyn HC. The attosecond nonlinear optics of bright coherent Xray generation. Nat Photonics. 2010;4(12):822-32. https://doi.org/10.1038/nphoton.2010.256.

4. Sakdinawat A, Attwood D. Nanoscale X-ray imaging. Nat Photonics. 2010:4(12):840-8. https://doi.org/10.1038/nphoton.2 010.267 .

5. Chang Z, Corkum PB, Leone SR. Attosecond optics and technology: progress to date and future prospects [invited]. J Opt Soc Am B. 2016;33(6):1081. https://doi.org/10.1364/JOSAB.33.001081.

6. Krausz F, Ivanov M. Attosecond physics. Rev Mod Phys. 2009;81(1):163-234. https://doi.org/10.1103/RevModPhys.81.163.

7. Constant E, Garzella D, Breger P, Mével E, Dorrer C, Le Blanc C, et al. Optimizing high harmonic generation in absorbing gases: model and experiment. Phys Rev Lett. 1999;82(8):1668-71. https://doi.org/10.1103/PhysRevLett.82.1668.

8. Takahashi E, Nabekawa Y, Midorikawa K. Generation of 10- $\mu \mathrm{J}$ coherent extreme-ultraviolet light by use of high-order harmonics. Opt Lett. 2002;27(21):1920. https://doi.org/10.1364/OL.27.001920.

9. Lee J, Carlson DR, Jones RJ. Optimizing intracavity high harmonic generation for XUV fs frequency combs. Opt Express. 2011;19(23):23315-26. https://doi.org/10.1364/OE.19.023315.

10. Cingöz A, Yost DC, Allison TK, Ruehl A, Fermann ME, Hartl I, et al. Direct frequency comb spectroscopy in the extreme ultraviolet. Nature. 2012;482(7383):68-71. https://doi.org/10.1038/nature10711. 
11. Hädrich S, Klenke A, Rothhardt J, Krebs M, Hoffmann A, Pronin O, et al. High photon flux table-top coherent extremeultraviolet source. Nat Photonics. 2014;8(10):779-83. https://doi.org/10.1038/nphoton.2014.214.

12. Porat G, Heyl CM, Schoun SB, Benko C, Dörre N, Corwin KL, et al. Phase-matched extreme-ultraviolet frequency-comb generation. Nat Photonics. 2018;12(7):387-91. https://doi.org/10.1038/s41566-018-0199-z.

13. Klas R, Demmler S, Tschernajew M, Hädrich S, Shamir Y, Tünnermann A, et al. Table-top milliwatt-class extreme ultraviolet high harmonic light source. Optica. 2016;3(11):1167. https://doi.org/10.1364/OPTICA.3.001167.

14. Comby A, Descamps D, Beauvarlet S, Gonzalez A, Guichard F, Petit S, et al. Cascaded harmonic generation from a fiber laser: a milliwatt XUV source. Opt Express. 2019;27(15):20383-96. https://doi.org/10.1364/OE.27.020383.

15. Wang H, Xu Y, Ulonska S, Robinson JS, Ranitovic P, Kaindl RA. Bright high-repetition-rate source of narrowband extremeultraviolet harmonics beyond $22 \mathrm{eV}$. Nat Commun. 2015;6(1):7459. https://doi.org/10.1038/ncomms8459.

16. Saraceno CJ, Sutter D, Metzger T, Abdou AM. The amazing progress of high-power ultrafast thin-disk lasers. J Eur Opt Soc Publ. 2019;15(1):15. https://doi.org/10.1186/s41476-019-0108-1.

17. Keunecke M, Möller C, Schmitt D, Nolte H, Jansen GSM, Reutzel M, et al. Time-resolved momentum microscopy with a $1 \mathrm{MHz}$ high-harmonic extreme ultraviolet beamline. Rev Sci Instrum. 2020;91(6):063905. https://doi.org/10.1 063/5.0006531.

18. Comby A, Bloch E, Beauvarlet S, Rajak D, Beaulieu S, Descamps D, et al. Bright, polarization-tunable high repetition rate extreme ultraviolet beamline for coincidence electron-ion imaging. J Phys B Atomic Mol Phys. 2020;53(23):234003. https://doi.org/10.1088/1361-6455/abbe27.

19. Geneaux R, Marroux HJB, Guggenmos A, Neumark DM, Leone SR. Transient absorption spectroscopy using high harmonic generation: a review of ultrafast X-ray dynamics in molecules and solids. Philos Trans R Soc A Math Phys Eng Sci. 2019;377(2145):20170463. https://doi.org/10.1098/rsta.2017.0463.

20. Hütten K, Mittermair M, Stock SO, Beerwerth R, Shirvanyan V, Riemensberger J, et al. Ultrafast quantum control of ionization dynamics in krypton. Nat Commun. 2018;9(1):719. https://doi.org/10.1038/s41467-018-03122-1.

21. Kfir O, Zayko S, Nolte C, Sivis M, Möller M, Hebler B, et al. Nanoscale magnetic imaging using circularly polarized highharmonic radiation. Sci Adv. 2017;3:eaao4641. https://doi.org/10.1126/sciadv.aao4641.

22. LaForge AC, Benediktovitch A, Sukharnikov V, Krušič Š, Žitnik M, Debatin M, et al. Time-resolved quantum beats in the fluorescence of helium resonantly excited by XUV radiation. J Phys B Atomic Mol Phys. 2020;53(24):244012. https://doi. org/10.1088/1361-6455/abc660

23. Rothhardt J, Bilal M, Beerwerth R, Volotka AV, Hilbert V, Stöhlker T, et al. Lifetime measurements of ultrashort-lived excited states in be-like ions. X-Ray Spectrom. 2020;49(1):165-8. https://doi.org/10.1002/xrs.3079.

24. González-Castrillo A, Martín F, Palacios A. Quantum state holography to reconstruct the molecular wave packet using an attosecond XUV-XUV pump-probe technique. Sci Rep. 2020;10(1):12981. https://doi.org/10.1038/s41598-020-69733-1.

25. Corkum PB, Krausz F. Attosecond science. Nat Phys. 2007;3(6):381-7. https://doi.org/10.1038/nphys620.

26. Lépine F, Ivanov MY, Vrakking MJJ. Attosecond molecular dynamics: fact or fiction? Nat Photonics. 2014;8(3):195-204. https://doi.org/10.1038/nphoton.2014.25.

27. Siegrist F, Gessner JA, Ossiander M, Denker C, Chang Y-P, Schröder MC, et al. Light-wave dynamic control of magnetism. Nature. 2019;571(7764):240-4. https://doi.org/10.1038/s41586-019-1333-x.

28. Müller M, Kienel M, Klenke A, Gottschall T, Shestaev E, Plötner M, et al. $1 \mathrm{~kW} 1 \mathrm{~mJ}$ eight-channel ultrafast fiber laser. Opt Lett. 2016;41(15):3439-42. https://doi.org/10.1364/OL.41.003439.

29. Shiner AD, Trallero-Herrero C, Kajumba N, Bandulet H-C, Comtois D, Légaré F, et al. Wavelength scaling of high harmonic generation efficiency. Phys Rev Lett. 2009;103(7):073902. https://doi.org/10.1103/PhysRevLett.1 03.073902.

30. Ishikawa KL, SchiessI K, Persson E, Burgdörfer J. Fine-scale oscillations in the wavelength and intensity dependence of highorder harmonic generation: connection with channel closings. Phys Rev A. 2009;79(3):033411. https://doi.org/10.1103/ PhysRevA.79.033411.

31. Zaïr A, Holler M, Guandalini A, Schapper F, Biegert J, Gallmann L, et al. Quantum path interferences in high-order harmonic generation. Phys Rev Lett. 2008;100(14):143902. https://doi.org/10.1103/PhysRevLett.100.143902.

32. Lewenstein M, Balcou P, Ivanov MY, L'Huillier A, Corkum PB. Theory of high-harmonic generation by low-frequency laser fields. Phys Rev A. 1994;49(3):2117-32. https://doi.org/10.1103/PhysRevA.49.2117.

33. Descamps D, Guichard F, Petit S, Beauvarlet S, Comby A, Lavenu L, et al. High-power sub-15 fs nonlinear pulse compression at $515 \mathrm{~nm}$ of an ultrafast Yb-doped fiber amplifier. Opt Lett. 2021;46:1804. https://oi.org/10.1364/OL.419683.

34. Xia J, Altucci C, Amoruso S, Bruzzese R, Velotta R, Wang X. Generation of high energy, $30 \mathrm{fs}$ pulses at $527 \mathrm{~nm}$ by hollowfiber compression technique. Opt Express. 2008;16(6):3527-36. https://doi.org/10.1364/OE.16.003527.

35. Pronin O, Pervak V, Fill E, Rauschenberger J, Krausz F, Apolonski A. Ultrabroadband efficient intracavity XUV output coupler. Opt Express. 2011;19(11):10232-40. https://doi.org/10.1364/OE.19.010232.

36. Klas R, Kirsche A, Tschernajew M, Rothhardt J, Limpert J. Annular beam driven high harmonic generation for high flux coherent XUV and soft X-ray radiation. Opt Express. 2018;26(15):19318-27. https://doi.org/10.1364/OE.26.019318.

37. Hädrich S, Rothhardt J, Demmler S, Tschernajew M, Hoffmann A, Krebs M, et al. Scalability of components for kW-level average power few-cycle lasers. Appl Opt. 2016:55(7):1636-40. https://doi.org/10.1364/AO.55.001636.

38. Rothhardt J, Rothhardt C, Müller M, Klenke A, Kienel M, Demmler S, et al. $100 \mathrm{~W}$ average power femtosecond laser at 343 nm. Opt Lett. 2016;41(8):1885-8. https://doi.org/10.1364/OL.41.001885.

39. Travers JC, Chang W, Nold J, Joly NY, PSJ R. Ultrafast nonlinear optics in gas-filled hollow-core photonic crystal fibers [Invited]. J Opt Soc Am B. 2011;28:A11. https://doi.org/10.1364/JOSAB.28.000A11.

40. Ammosov MV, Delone NB, Krainov VP. In: Alcock JA, editor. Sov. Phys. JETP Tunnel ionization of complex atoms and atomic ions in electromagnetic field; 1986. p. 138. https://doi.org/10.1117/12.938695.

41. Henke BL, Gullikson EM, Davis JC. X-ray interactions: Photoabsorption, scattering, transmission, and reflection at E = 5030,000 eV, Z = 1-92. At Data Nucl Data Tables. 1993;54(2):181-342. https://doi.org/10.1006/adnd.1993.1013.

\section{Publisher's Note}

Springer Nature remains neutral with regard to jurisdictional claims in published maps and institutional affiliations. 